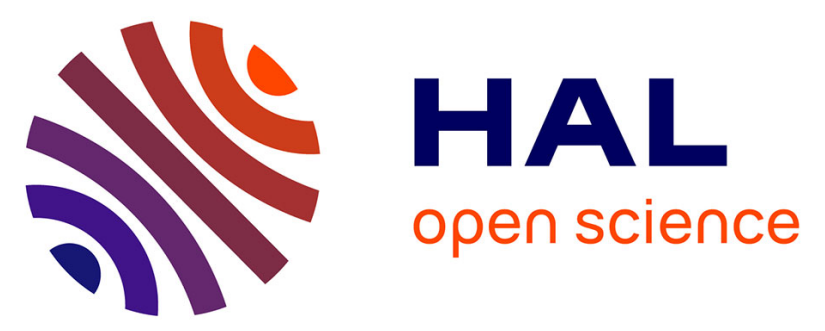

\title{
Serum resistance and phase variation of a nasopharyngeal non-typeable Haemophilus influenzae isolate
}

Sabine Lichtenegger, Isabelle Bina, Sanel Durakovic, Philippe Glaser, Sarah Tutz, Stefan Schild, Joachim Reidl

\section{To cite this version:}

Sabine Lichtenegger, Isabelle Bina, Sanel Durakovic, Philippe Glaser, Sarah Tutz, et al.. Serum resistance and phase variation of a nasopharyngeal non-typeable Haemophilus influenzae isolate. International Journal of Medical Microbiology, 2017, 307 (2), pp.139-146. 10.1016/j.ijmm.2017.01.005 . pasteur-03107776

\section{HAL Id: pasteur-03107776}

https://hal-pasteur.archives-ouvertes.fr/pasteur-03107776

Submitted on 12 Jan 2021

HAL is a multi-disciplinary open access archive for the deposit and dissemination of scientific research documents, whether they are published or not. The documents may come from teaching and research institutions in France or abroad, or from public or private research centers.
L'archive ouverte pluridisciplinaire HAL, est destinée au dépôt et à la diffusion de documents scientifiques de niveau recherche, publiés ou non, émanant des établissements d'enseignement et de recherche français ou étrangers, des laboratoires publics ou privés.

\section{(ㅇ)(1) $\$$}

Distributed under a Creative Commons Attribution - NonCommercial - NoDerivatives 44.0 
Title:

Serum resistance and phase variation of a nasopharyngeal non-typeable Haemophilus influenzae isolate

Sabine Lichtenegger ${ }^{1,2} *^{\#}$, Isabelle Bina $^{1}{ }^{*}$, Sanel Durakovic ${ }^{1}$, Philippe Glaser $^{3}$, Sarah Tutz ${ }^{1}$, Stefan Schild $^{1 \mathrm{a}}$, and Joachim Reidl ${ }^{1 \# \mathrm{a}}$

${ }^{1}$ Institute of Molecular Biosciences, University of Graz, Humboldtstr. 50, 8010 Graz, Austria, aBioTechMed-Graz, Austria

${ }^{2}$ Institute of Hygiene, Microbiology and Environmental Medicine, Medical University Graz, Neue Stiftingtalstraße 2, 8010 Graz, Austria,

${ }^{3}$ Insitut Pasteur, 25-28 rue du docteur Roux, 75015 Paris, France

KEYWORDS: serum resistance, lpsA, Haemophilus influenzae, phase variation.

* Contributed equally

\#Addresses to shared corresponding authors:

Joachim Reidl, joachim.reidl@uni-graz.at

Institute of Molecular Biosciences

University of Graz, Humboldtstrasse 50,

8010 Graz, Austria

Tel.: 00433163801978

Fax.: 00433163809019

Sabine Lichtenegger, sabine.lichtenegger@medunigraz.at Institute of Hygiene, Microbiology and Environmental Medicine Medical University Graz 8010 Graz, Austria

Tel.: 004331638573603

Fax.: 004331638579637 


\section{ABSTRACT}

Haemophilus influenzae harbours a complex array of factors to resist human complement attack. As non-typeable $H$. influenzae (NTHi) strains do not possess a capsule, their serum resistance mainly depends on other mechanisms including LOS decoration. In this report, we describe the identification of a highly serum resistant, nasopharyngeal isolate (NTHi23) by screening a collection of 77 clinical isolates. For NTHi23, we defined the MLST sequence type 1133, which matches the profile of a previously published invasive NTHi isolate. A detailed genetic analysis revealed that NTHi23 shares several complement evading mechanisms with invasive disease isolates. These mechanisms include the functional expression of a retrograde phospholipid trafficking system and the presumable decoration of the LOS structure with sialic acid. By screening the NTHi23 population for spontaneous decreased serum resistance, we identified a clone, which was about $10^{3}$-fold more sensitive to complement-mediated killing. Genome-wide analysis of this isolate revealed a phase variation in the $\mathrm{N}^{\prime}$-terminal region of $\operatorname{lps} A$, leading to a truncated version of the glycosyltransferase (LpsA). We further showed that a lpsA NTHi23 knockout mutant exhibits a decreased invasion rate into human alveolar basal epithelial cells. Since only a small proportion of the NTHi23 population expressed the serum sensitive phenotype, resulting from $\operatorname{lps} A$ phase-off, we conclude that the nasopharyngeal environment selected for a population expressing the intact and functional glycosyltransferase. 


\section{INTRODUCTION}

The Gram-negative bacterium Haemophilus influenzae is a human-specific pathogen, which can be subdivided into encapsulated and non-encapsulated strains. The latter are usually referred to as nontypeable H. influenzae (NTHi) strains (Pittman, 1931). Encapsulated H. influenzae strains, foremost of type b capsule (Hib), are responsible for a variety of severe invasive diseases, and are mostly observed in infants and children, causing epiglottitis, sepsis, and meningitis. After the introduction of an effective vaccination strategy against type b capsule strains in the early 1990s, invasive diseases decreased substantially in populations in which the vaccine was applied (Kelly et al., 2004; Ulanova, 2013). Since Hib-vaccine is type b capsule specific, it does not protect against NTHi caused diseases. NTHi strains are mostly assigned to the commensals of the respiratory tract, and are mainly producing local disease in the upper and lower respiratory tract, such as acute otitis media, sinusitis, and bronchitis. Additionally, they cause exacerbations in chronic diseases such as asthma and chronic obstructive pulmonary disease (COPD). Since the introduction of the Hib vaccine and the resulting decrease in Hib infections, more attention was attracted by NTHi strains. Thereby, genotypic and phenotypic differences were characterized trying to define clades of invasive isolates (Erwin et al., 2005; Erwin et al., 2008). Increasing numbers of reports indicate that NTHi cause invasive diseases, which are as serious as those caused by encapsulated strains and which occur in infants, children and in adults. Hence, there is evidence for a shift from invasive diseases caused by encapsulated strains to those caused by NTHi infections as monitored in vaccinated areas (Giufre et al., 2011; Rubach et al., 2011).

Human pathogens, including $H$. influenzae, need to constantly cope with the various defence mechanisms of the immune system. H. influenzae is susceptible to complement mediated killing and in order to infect its human host it has to counteract this otherwise lethal attack (Zola et al., 2009). 
$H$. influenzae strains have developed several sophisticated strategies to evade the complement system. The genetic locus of the polysaccharide capsule of typeable strains was shown to be frequently amplified in clinical isolates, which was associated with decreased complement mediated killing (Noel et al., 1996). Furthermore, diverse outer membrane proteins were shown to be involved in complement resistance. In 2014, Rosadini et al. identified outer membrane protein P5 as an essential factor in serum resistance. They showed that P5 decreases binding of IgM antibodies and thereby is interfering with classical complement activation. Additionally, P5 can bind factor $\mathrm{H}$, which inhibits the alternative pathway of complement-mediated lysis (Rosadini et al., 2014). Another outer membrane protein, namely adhesion protein E (PE), was shown to bind vitronectin, an important inhibitor of the membrane attack complex. Thus, $H$. influenzae lacking PE was rendered more susceptible towards complement mediated killing (Hallstrom et al., 2009). This implicates another strategy employed by $H$. influenzae, which is attracting complement regulatory factors to hinder the complement cascade to proceed. In addition to binding factor $\mathrm{H}$ and vitronectin (Hallstrom et al., 2009; Hallstrom et al., 2006; Hallstrom et al., 2008), NTHi decrease classical complement activation by sequestering C4b binding protein (Hallstrom et al., 2007).

Furthermore, phase variable LOS structures have been shown to play a major role in resistance to the classical as well as the alternative pathway of complement activation. In 1993, High et al. identified lic $2 A$ as a phase-variable gene, facilitating the Gal(1-4)beta Gal extension to the LOS structure (High et al., 1993) Not only lic $2 A$ but also $\lg t C$ and $\operatorname{lex} 2 A$ were shown to switch from phase-off to phase-on during passage through human serum. LgtC as well as Lex2A independently contribute to better survival by shielding conserved LOS inner core structures (Clark et al., 2013). Due to the phase variability of LOS, a distinct $H$. influenzae population comprises different phase variants with different structures and modifications. The respective host condition might select for a modification-specific population while clearing the other. The phase variable structure phosphorylcholine (ChoP) represents one example, where one environment selects for phase-on while another environment selects for phase-off (Weiser et al., 1998). ChoP is recognized by C- 
reactive protein (CRP), which in turn leads to the activation of the complement system. Environments with high levels of CRP select for licl phase-off variants, which lack the ChoP moiety (Clark et al., 2012; Tong et al., 2000; Weiser et al., 1998). In other environments this modification seems to be advantageous. Therefore, the phase variability of the lic 1 locus provides an efficient mean to frequently turn off and on gene expression, depending on the environment $H$. influenzae faces. This is also suggested for other phase variable loci, which are associated with high mutation rates and will need further investigations (Clark et al., 2013).

As surveillance programs report a steady increase in invasive disease due to non-typeable strains (Giufre et al., 2011; Rubach et al., 2011), we conducted a detailed analysis of a strain collection consisting of invasive and non-invasive isolates with regards to their serum resistance profile. Our findings highlight that there is no observable correlation between serum resistance and invasiveness. Interestingly, our studies identified a highly serum resistant isolate (NTHi23), which was derived from a healthy donor during a routine examination. In addition, we revealed that the high serum resistance of the isolate depends on the phase variable gene lpsA, involved in LOS synthesis and enhanced invasion into epithelial cells. 


\section{MATERIALS AND METHODS}

Ethics statement. Human blood was obtained from five healthy adults in accordance with the Ethical Review Committee of the University of Graz (39/31/63 ex 2012/13). All subjects provided written consent.

Culturing methods and growth conditions. Clinical NTHi isolates used during this study were obtained from AGES-Graz (Agentur Gesundheit und Ernährungssicherheit / Zentrum fuer lebensmittelbedingte Infektionskrankheiten) and the Institute for Hygiene and Microbiology (University of Würzburg, Germany). 46 strains are classified as invasive and 31 as non-invasive isolates. Among them, NTHi23 was isolated from a 6 year-old female during routine examination (personal communication Dr. Georg Steindl, AGES Graz) and was analysed in detail. All generated, isogenic mutants are listed in Table 1. H. influenzae strains were grown aerobically at $37^{\circ} \mathrm{C}$ in brain heart infusion (BHI) supplemented with $10 \mu \mathrm{g} / \mathrm{ml} \mathrm{NAD}$ and hemin-solution, containing $20 \mu \mathrm{g} / \mathrm{ml}$ hemin, $20 \mu \mathrm{g} / \mathrm{ml}$ L-histidine and $0.08 \%$ triethanolamine (sBHI). To select for chloramphenicol $^{\mathrm{r}}(\mathrm{Cm}, 2 \mu \mathrm{g} / \mathrm{ml})$ or $\operatorname{kanamycin}^{\mathrm{r}}(\mathrm{Km}, 50 \mu \mathrm{g} / \mathrm{ml})$ corresponding resistance gene cassettes were applied (see below). A549 cells (human alveolar lung adenocarcinoma cells; ATCC CCL87) were cultured in DMEM supplemented with $10 \%$ fetal calf serum at $37^{\circ} \mathrm{C}$ with $5 \% \mathrm{CO}_{2}$.

Construction of deletion mutants. Deletion mutants were constructed as previously described (Lichtenegger et al., 2014), using an overlap extension PCR. Briefly, the target gene was exchanged by a $\mathrm{Cm}$ or $\mathrm{Km}$ resistance cassette obtained from plasmid pAKcat (Kraiss et al., 1998) or pKan $\pi$ (Oka et al., 1981), respectively. To amplify the three fragments, six oligonucleotide primers (x_1 x_6) were applied with $\mathrm{x}$ corresponding to the gene of interest. The upstream fragment was generated using $x \_1$ and $x \_2$, the antibiotic resistance cassette using $x \_3$ and $x \_4$ and the downstream fragment using $\mathrm{x} \_5$ and $\mathrm{x} \_6$. Oligonucleotides are listed in Table 2. The final PCR fragment was transformed into strain NTHi23, applying a modification of the static aerobic method established by Gromkova et al. (Gromkova et al., 1989). One single colony was suspended in 200 
$\mu \mathrm{l}$ sBHI (supplemented BHI medium) and incubated with DNA at $30^{\circ} \mathrm{C}$ overnight. Positive transformants were selected using agar plates containing the appropriate antibiotic.

IpsA complementation. The lpsA mutation was chromosomally restored according to a previously described method (Lichtenegger et al., 2014). The lpsA gene was fused to a Km resistance cassette by overlap extension PCR and the final fragment was transformed into NTHi23 $\Delta$ lpsA by the above-described transformation procedure.

Serum bactericidal assays. Bactericidal assays were carried out as previously described (Lichtenegger et al., 2014). Serum from 5 healthy volunteers served as a source of antibodies and complement factors. Cells were grown to mid-log phase under shaking conditions at $180 \mathrm{rpm}$ and transferred to Hank's buffer. Normal human serum was applied in concentrations varying from 3$35 \%$. After incubation for $45 \mathrm{~min}$ at $37^{\circ} \mathrm{C}$ with rotation, cells were plated on sBHI to determine viable cell counts. In parallel either a non-serum or heat-inactivated control was performed. Heatinactivated serum was generated at $56^{\circ} \mathrm{C}$ for $30 \mathrm{~min}$.

High-throughput bactericidal assays. High-throughput assays were performed in 96 well polystyrene plates. Isolates were grown to mid-log phase in static liquid cultures. Mid-log phase cultures were shifted to Hank's buffer containing $20 \%$ of human serum. After incubation at $37^{\circ} \mathrm{C}$ for 45 min alamarBlue cell viability reagent array was added to detect metabolic activity, indicating viable and metabolic active bacterial cells. Viable cells reduce the active component of alamarBlue (resazurin) to resorufin, which is fluorescent. Fluorescence was monitored $2 \mathrm{~h}$ after addition of the reagent in a plate-based fluorescence spectrometer (FLUOstar, OMEGA, BMG Labtech). The fluorescence signal, we observed for the heat-inactivated serum control, was set to $100 \%$.

Invasion assay. Adhesion and invasion assays were performed as previously described (Tchoupa et al., 2015). 24 hours prior to infection A549 cells were seeded in 12 well plates at a density of $3 \times$ $10^{5}$ cells per well. Cells were infected with $H$. influenzae NTHi23 wt strain or the lpsA mutant at a 
multiplicity of infection (MOI) of 100. Two hours after infection the bacteria containing medium was replaced by cell culture medium containing $100 \mu \mathrm{g} / \mathrm{ml}$ gentamicin to efficiently kill extracellular bacteria. After a 1 hour incubation step, the number of intracellular bacteria was determined. Consequently, A549 cells were lysed using PBS containing 0.5\% saponin for 15 minutes. To determine viable bacterial cell counts, the lysates were plated on sBHI agar plates at the appropriate dilutions. The number of internalized bacteria was normalized to the bacterial input. Adhesion assay. Adhesion assays were performed in parallel to the invasion assay and according to (Voges et al., 2012). Consequently, A549 cells were seeded 24 hours prior to infection at a cell density of $3 \times 10^{5}$ cells per well. Cells were infected with $H$. influenzae NTHi23 wt strain or the IpsA mutant at a multiplicity of infection (MOI) of 100. Two hours after infection the bacteria containing supernatant was removed and cells were washed twice with PBS and lysed with PBS containing $0.5 \%$ saponin for 15 minutes. To determine viable bacterial cell counts, the lysates were plated on sBHI agar plates at the appropriate dilutions. The number of adherent bacteria was calculated by subtracting the number of intracellular bacteria from the in parallel-performed invasion assay from the number of viable bacteria determined during the adhesion assay. The number of adherent bacteria was normalized to the bacterial input.

MLST analysis. MLST analysis was performed according to the MLST $H$. influenzae database (http://haemophilus.mlst.net). Briefly, defined fragments of seven $H$. influenzae housekeeping genes, namely $a d k, a t p G, f r d B, f u c K, m d h$, pgi and $r e c A$, were amplified with the oligonucleotides listed in Table 2. After sequencing the amplified fragments (Table S1), the encoding gene sequences were entered in the MLST database and the allele number for each gene was obtained. The MLST database provides a sequence type in consequence of the allele profile and subsequently enables you to identify genetic matches.

Genome sequencing. NTHi23 and NTHi23_variant genome sequences were determined by using the Illumina technology. Libraries were constructed by using the Illumina Nextera XT kit. Sequencing was performed on an Illumina MiSeq, 140 bases single read run. Sequences were 
assembled with Velvet (DOI 10.1101/gr.074492.107) and an optimized K value. Differences (SNP and short INDELS) between the two isolates were determined by aligning reads of strains NTHi23_variant on the contigs of strain NTHi23 using BRESEQ (DOI 10.1007/978-1-4939-05546_12). Contig sequences of strain NTHi23 were deposited at EMBL (Genome project pending). 


\section{RESULTS AND DISCUSSION}

Serum resistance does not coincide with invasiveness. Although non-typeable $H$. influenzae strains usually cause mucosal infections, they are increasingly reported to cause invasive disease (Giufre et al., 2011; Rubach et al., 2011). In general, serum resistance is suggested to facilitate the spread of bacterial pathogens in the human body. In the present study we investigated 77 clinical NTHi strains, isolated from various infection sites, for their ability to survive in $20 \%$ human serum. The isolates were collected from patients suffering from various diseases (lymphadenitis, meningitis, pneumonia, mastoiditis, endophthalmitis, sepsis, and sinusitis). We blotted and compared the data for invasive $(n=46)$ and non-invasive obtained isolates $(n=31)$ Figure 1 to elucidate a potential correlation between serum resistance and invasiveness. As there was no significant difference between the two groups, the hypothesis of a correlation between serum resistance and invasiveness cannot be confirmed. These results are in agreement with a study by Hallström et al., which suggests that serum resistance is equally important for strains causing upper respiratory tract infections and strains causing sepsis. Nevertheless, they found that serum resistance correlates with the severity of the disease and might therefore become increasingly important once the infection is established (Hallstrom and Riesbeck, 2010). We extend and support this analysis by characterizing NTHi strains from multiple infection sites and conclude that high serum resistance is not an exclusive marker of invasive strains.

\section{An NTHi isolate displays a similar serum resistance profile when compared to an} encapsulated strain. In the course of the performed high-throughput assay (see Material and Methods), we identified a recognizable highly serum resistant strain NTHi23, which was isolated from the nasopharynx of an asymptomatic 6-years old female patient. In Figure 2 we compare the serum survival rate of NTHi23 and the well-characterized type b strain, Hib Eagan (Noel et al., 1996; Sutton et al., 1982). The polysaccharide capsule of the typeable strain represents an important 
protective coat against complement-mediated attack (Cerquetti et al., 2005; Corn et al., 1993). Although, the obtained survival data between the two strains show no significant differences, NTHi23 tends to display even higher survival in $35 \%$ human serum than the type $b$ strain. Therefore, these findings provide further evidence that NTHi strains have developed sophisticated strategies for complement evasion, compensating for the lack of a capsule.

\section{MLST analysis reveals an exact sequence type match of NTHi23 with a previously identified}

disease isolate. Highly serum resistant NTHi strains, although having the potential to cause disease, also persist in a commensal state (Hallstrom et al., 2010). To further unravel the nature and origin of NTHi23, we performed an MLST analysis (Table S1). This analysis revealed that NTHi23 displays an exact sequence type match with NTHi strain Hi391, isolated from a patient suffering from invasive disease (Giufre et al., 2011). This finding supports the hypothesis, that host factors play a prominent role in the outcome of a $H$. influenzae infection. According to this hypothesis, a similar isolate can reside as a commensal in the nasopharynx of one patient, while having the potential to cause severe disease in another patient. NTHi strains are more likely to cause disease in immunocompromised patients. However, an invasive outcome of an $H$. influenzae infection not always correlates with the immune status of the human carrier (Hallstrom et al., 2010). This indicates that other factors are involved in the clinical phenotype of an infection. These factors might include virulence factors of the particular strain and potentially pre-existing antibodies of the host due to prior contact to other H. influenzae strains (Hallstrom et al., 2010). Taken together, this analysis strengthens the hypothesis that the invasiveness and virulence of a particular strain relies on a complex array of bacterial as well as host factors. 
Pathways, previously associated with serum resistance, play a crucial role in the serum resistant phenotype of strain NTHi23. Several mechanisms and pathways contributing to serum resistance were unravelled recently. These mechanisms include the incorporation of sialic acid into the LOS structure (Figueira et al., 2007), the overexpression of a retrograde phospholipid trafficking system (Malinverni and Silhavy, 2009; Nakamura et al., 2011) and the expression of the hypothetical protein Hi0461 (Langereis et al., 2012; Nizet et al., 1996). In order to obtain a comprehensive overview of serum resistance mechanisms for NTHi23 a detailed characterization was conducted.

H. influenzae lacks the ability to de novo synthesize sialic acid and therefore relies on the uptake of external sources (Severi et al., 2005). A deletion of siaP inactivates the tripartite sialic acid transport system (SiaPQM) and subsequently sialic acid attachment to the LOS structure. LOS modification with sialic acid has been attributed with a critical role in resistance to complement mediated killing by the alternative pathway (Figueira et al., 2007). Figure 3 shows that a siaPmutant displays decreased survival in $20 \%$ human serum, when compared to the wt strain. This finding shows that SiaP function plays a vital role in the serum resistance of strain NTHi23.

In addition to LOS decoration with sialic acid, LOS compactness, which is influenced by the $\mathrm{VacJ} / \mathrm{Yrb} \mathrm{ABC}$ transport system, impacts the serum resistance of NTHi23. Not only a siaP-mutant, but also an $y r b E$ - mutant shows decreased serum resistance (Figure 3) in this strain background. YrbE is part of a retrograde phospholipid trafficking system, comprising at least six proteins: VacJ, YrbC, YrbD, YrbE, YrbB, YrbF. The model for its involvement in serum resistance suggests that blocking the lipid trafficking system leads to an accumulation of phospholipids in the outer membrane and subsequently to the destabilization of intermolecular LOS interactions. This in turn exposes epitopes, which are recognized by bactericidal antibodies, leading to antibody-mediated killing. Strains, which were isolated from lower respiratory tract infections display increased expression of VacJ and YrbE (Malinverni and Silhavy, 2009; Nakamura et al., 2011). The observation that a $y r b E$ - mutant is more susceptible to complement attack in NTHi23 suggests that 
also in this strain LOS epitopes are recognized by NHS antibodies upon an alteration of the outer membrane phospholipids:LOS ratio.

As evident from Figure 3, a Hi0461- mutant also displays decreased serum resistance in the NTHi23 background. As shown previously, a deletion of the R2866_0112 gene, the homolog of Rd KW-20 Hi0461, leads to increased IgM binding and subsequently to an increase in serum sensitivity (Langereis et al., 2012). R2866_0112 mutants express various numbers of truncated LOS glycoforms. Furthermore, a prominent glycoform is absent and therefore it is tempting to suggest that R2866_0112/ HiO461 does not encode a transferase, which is directly involved in LOS biosynthesis (Langereis et al., 2012; Nizet et al., 1996).

In conclusion, our data demonstrate that the described mechanisms for serum resistance can be extended to our strain, strengthening the hypothesis that the underlying mechanisms can be considered general evasion strategies of $H$. influenzae. In addition the characterization of the three single mutants provides a first insight into the serum resistance mechanisms of strain NTHi23.

A subpopulation of the NTHi23 population displays altered serum resistance. Clark and colleagues hypothesized that bacteria evade killing by the host immune system through selective enrichment of resistant bacteria from a mixed population (Clark et al., 2013). Assuming that the isolated NTHi23 population also displays differences in their serum resistance status, we hypothesized that individual clones would express differences in their survival when incubated in human serum. The identification of more serum susceptible variants could contribute to unravel mechanisms involved in the serum resistance of the investigated NTHi strain. Therefore, 60 individual clones were tested in a high-throughput assay for their survival in human serum. In the course of this study, we identified a variant of the strain (NTHi23_variant), which was nearly 1000fold more sensitive than the mixed population. To validate the obtained results, we tested the variant in our standar serum assay, which confirmed the serum sensitive phenotype (Figure 4). 
These results show that variations in serum resistance levels exist within the NTHi23 population. The observation, that only one out of 60 shows decreased serum resistance suggests that the in vivo situation, in this case the nasopharynx of a child, selected for serum resistant variants.

The NTHi23 population is enriched in $\operatorname{lps} A$ phase-on. To determine the genetic basis of the serum sensitive phenotype of NTHi23_variant, the whole genome of the wt and the variant strain was sequenced. The sequence analysis revealed a 5 base insertion (GCACA) in lpsA. LpsA belongs to the family of GT-A glycosyltransferases and mediates the transfer of the initial hexose to Hep (Deadman et al., 2006). Interestingly, NTHi23 displays a short sequence repeat (5 nts per repeat) at the $5^{\prime}$ end of the lpsA coding region. A seven repeat unit in strain NTHi23 leads to the expression of a functional protein. The five base pair insertion leads to a frame shift mutation in $\operatorname{lps} A$ and subsequently to the formation of a preliminary stop codon and the expression of a truncated, most likely non-functional protein. Phase variations, resulting from slipped-strand mispairing, are frequently found in genes encoding LOS modifications, but were not comprehensively described for the lpsA gene.

To validate and investigate the observed $\operatorname{lps} A$ phenotype, we constructed a deletion mutant and complementation strain for the phase variable gene. Figure 5 shows that the deletion strain displays similar serum sensitivity as observed for the phase-off variant. Reintroducing the phase-on variant of the lpsA gene in the deletion mutant complemented serum sensitivity. Collectively these data suggest that the serum sensitive phenotype of NTHi23_variant can presumably be attributed solely to the expression of a truncated version of LpsA.

The NTHi23 $\Delta l p s A$ mutant displays decreased invasion of epithelial cells. NTHi23 was isolated from a 6-year old carrier showing no signs of disease. To further determine the biological relevance of the $\operatorname{lps} A$ phase variation and the fact that the phase-on variant was predominantly represented in 
the isolated NTHi23 population, we performed an invasion and adhesion assay. The wt and the $\operatorname{lps} A$ mutant adhered equally well to epithelial cells (Figure 6A). In contrast, the lpsA mutant was significantly attenuated in cell invasion (Figure 6B). This observation correlates with the observed serum sensitive phenotype of $\operatorname{lps}$. The results of both assays might shed light on the in vivo selective pressure towards the $\operatorname{lps} A$ phase-on variant in the nasopharynx of the human host. Whether the lpsA associated modification of the LOS structure plays a role in receptor mediated internalization into human epithelial cells remains elusive and needs further investigation.

At least four alleles of $\operatorname{lps} A$ exist with the presence of one excluding the presence of the others. Differences in the amino acid sequence, encoded by the different alleles, determine whether a Glc or Gal is added in a B1-2 or B1-3 linkage. One single amino acid residue determines which hexose is added to Hep $\mathrm{III}$. Threonine leads to the addition of galactose whereas cysteine, alanine or methionine leads to the addition of a glucose moiety (Deadman et al., 2006). For NTHi23 the amino acid sequence predicts that $\operatorname{lps} A$ displays glucosyltransferase activity as a cysteine is found at the amino acid position, which determines the attached residue. The nature of the initial hexose,

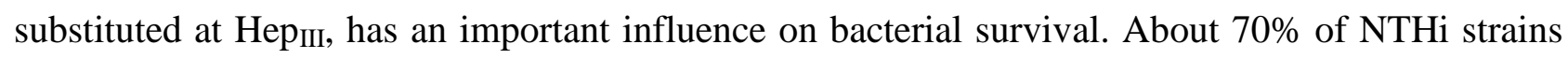
express HepIII-Glc, which is suggested to be recognized by naturally occurring IgM antibodies in turn leading to phagocytosis by human neutrophils (Langereis and Weiser, 2014). A Gal residue at this position, which is frequently found in the LOS of invasive strains, is not recognized by these IgM antibodies and seems therefore advantageous for invasive disease. Nevertheless, most of the strains investigated in the study by Langereis et al. express HepIII-Glc. Therefore, it is tempting to suggest that this structure, although recognized by naturally occurring antibodies, is advantageous in some microenvironments (Langereis and Weiser, 2014). A similar phenomenon is observed for the presence of ChoP in the LOS of $H$. influenzae strains, which leads to high serum resistance during nasopharyngeal colonization (Clark et al., 2012; Poole et al., 2013), but results in complement activation in the bloodstream due to binding of circulating CRP (Weiser et al., 1998). 
Additionally, HepIII-Glc is an acceptor for further extensions (e.g. the addition of a galactose moiety mediated by Lic2A), whereas Gal is suggested to be a terminal moiety. Additional substituents lead to shielding of the immune-dominant epitope HepIII-Glc and experimentally prevent IgM binding and phagocytosis (Langereis and Weiser, 2014). The fact that several USS sequences are located upstream and downstream of the lpsA gene leads to the assumption that this gene could be subject to horizontal gene transfer and might be readily exchanged between strains. This could represent an additional important tool to adapt to different niches within the human host as it is suggested that the addition of either a Glc or Gal potentially mediate survival in different niches (Langereis and Weiser, 2014).

The lpsA gene displays both intra- and interstrain variability. Intrastrain variation, which is defined as a difference in mother and progeny cells, arises from the phase variability of $\operatorname{lps} A$. According to a Nucleotide BLAST analysis, phase variable $\operatorname{lps} A$ seems to be found only in a small proportion of NTHi strains. This potentially arises from the fact that it represents a glycosyltransferase mediating the initial substitution of one of the conserved heptoses. Interstrain specificity results from the different alleles found in distinct strains. In conclusion, $\operatorname{lps} A$ plays a crucial role in the diversity and composition of the LOS molecule, which in turn mediates colonization efficiency and virulence.

Strain NTHi23 represents a highly serum resistant clinical isolate, which did not cause disease in a 6-year old female patient at the time of isolation. Nevertheless, it comprises several sophisticated strategies to cope with the immune system with LpsA presumably playing a crucial role. The phase-off switch in NTHi23_variant strengthens this hypothesis. Expressing a truncated LpsA protein, the NTHi23_variant most likely looses the ability to mediate LOS substitutions at Hep III. This subsequently leads to the accessibility of LOS core structures, which are suggested to be immune-dominant epitopes (Clark et al., 2013). In addition, the modification of the LOS core structure by LpsA seems to play a significant role in the internalization of NTHi23 in human epithelial cells. The lpsA phase-off strain (NTHi23_variant) represented just a small proportion of the population (one out of sixty). This observation suggests that the predominant phenotype (lpsA 
phase-on) was enriched during nasopharyngeal colonization. Interestingly, Poole and colleagues (Clark et al., 2012; Poole et al., 2013) monitored phase-variation during experimental colonization in the nasopharynx of healthy human volunteers with strain NTHi2019Str ${ }^{\mathrm{R}}$ over a several days period. They observed that a significant number of isolates switched, e.g. shown for licA and igaB from phase-off to phase-on during a 4-6 days period. The authors conclude that these genes might play a role in early nasopharynx colonization. This might be also the case for the lpsA gene, thereby contributing to an efficient nasopharyngeal colonization. These observations suggest that the nasopharynx of healthy subjects can provide an environment, which favours the selection of phaseon variants of various genes. In addition, we cannot exclude, that the child acquired the NTHi strain from a diseased person (family, presumable kindergarten or school).

Acknowledgements. Work was supported by the Austrian Science Fund (FWF) ERA-NET FWF I 662-B09 to SL, and JR. Clinical NTHi isolates were donated by AGES-Graz (Agentur Gesundheit und Ernährungssicherheit $\mathrm{GmbH} /$ Zentrum fuer lebensmittelbedingte Infektionskrankheiten, Dr. Georg Steindl, AGES Graz) and the Institute for Hygiene and Microbiology (Dr. Thien-Tri Lam, University of Würzburg, Germany) 
Table 1: Bacterial strains used

\begin{tabular}{|c|c|c|}
\hline & \multicolumn{2}{|c|}{ Genotype and Description $\quad$ Reference } \\
\hline Hib strain Eagan & $\begin{array}{l}\text { Encapsulated serotype b } \\
\text { strain obtained from A. } \\
\text { Wright }\end{array}$ & (Anderson et al., 1972) \\
\hline NTHi23 & $\begin{array}{l}\text { NTHi nasopharyngeal, } \\
\text { clinical isolate }\end{array}$ & $\begin{array}{l}\text { Agentur fuer Gesundheit } \\
\text { und Ernaehrungssicherheit } \\
\text { GmbH (AGES) }\end{array}$ \\
\hline NTHi23 $\Delta$ siaP & $\begin{array}{l}\text { siaP replaced with } \mathrm{Cm}^{\mathrm{R}} \\
\text { cassette }\end{array}$ & This study \\
\hline NTHi23 $\Delta y r b E$ & $\begin{array}{l}\text { yrbE replaced with } \mathrm{Cm}^{\mathrm{R}} \\
\text { cassette }\end{array}$ & This study \\
\hline NTHi23 $\Delta H i 0461$ & $\begin{array}{l}\mathrm{HiO461} \text { replaced with } \\
\mathrm{Km}^{\mathrm{R}} \text { cassette }\end{array}$ & This study \\
\hline NTHi23 $\Delta l p s A$ & $\begin{array}{l}\text { lpsA replaced with } \mathrm{Cm}^{\mathrm{R}} \\
\text { cassette }\end{array}$ & This study \\
\hline NTHi23 $\Delta l p s A$ lpsA+ & $\begin{array}{l}\text { chromsomally restored } \\
\text { lpsA } \mathrm{Km}^{\mathrm{R}}\end{array}$ & This study \\
\hline
\end{tabular}

Table 2: Oligonucleotides used

\begin{tabular}{lll}
\hline Primers & Sequences $\left(\mathbf{5} \mathbf{- 3}^{\mathbf{*}}\right.$ ) & Origin \\
\hline Mutagenesis & & This study \\
siaP_1 & GTATAACCTGACATCGTACTGC & This study \\
& ATTTAGTGTATGATGGTGTTTTTGAA & \\
siaP_3 & TTCGCTCCTGTGTGAACTTAT & This study \\
& ATAAGTTCACACAGGAGCGAATTCA & \\
siaP_4 & AAAACACCATCATACACTAAAT & This study \\
& GAACTTTTGCACTTCATAGAATTTTAG & \\
siaP_5 & GCGTTTAAGGGCACCAATAA & This study \\
& TTATTGGTGCCCTTAAACGCCTAAAA & \\
siaP_6 & TTCTATGAAGTGCAAAAGTTC & This study \\
\hline
\end{tabular}




\begin{tabular}{|c|c|c|}
\hline 0461_1 & ATCATACCAAGCAAATATTCTAAAC & This study \\
\hline \multirow[t]{2}{*}{ 0461_2 } & TCTTGTGCAATGTAACATCAGAGATTT & This study \\
\hline & TTCATTATTTTAGCCATTATCA & \\
\hline \multirow[t]{2}{*}{ 0461_3 } & TGATAATGGCTAAAATAATGAAAAAT & This study \\
\hline & CTCTGATGTTACATTGCACAAGA & \\
\hline \multirow[t]{2}{*}{ 0461_4 } & ACCCATTAACCAACATAAACATTGCGT & This study \\
\hline & GAAGAAGGTGTTGCTGAC & \\
\hline \multirow[t]{2}{*}{$0461 \_5$} & GTCAGCAACACCTTCTTCACGCAATGT & This study \\
\hline & TTATGTTGGTTAATGGGT & \\
\hline 0461_6 & AAAGCGAAAGAAAAACAAGGTAAAA & This study \\
\hline yrbE_1 & ATAAACGAGATATTAATGAATCAAAAT & $\begin{array}{l}\text { (Roier et } \\
\text { al., 2016) }\end{array}$ \\
\hline \multirow[t]{2}{*}{ yrbE_2 } & TGTATGATGGTGTTTTTGAGGTG & (Roier et \\
\hline & GATCATTCAAACAATTCCTTCACA & al., 2010) \\
\hline \multirow[t]{2}{*}{ yrbE_3 } & TGTGAAGGAATTGTTTGAATGAT & (Roier et \\
\hline & CСАССТСАAАAАCACCATCATACA & d1., 2 \\
\hline \multirow[t]{2}{*}{ yrbE_4 } & TTGTTTGTCTCATAAAAAAATCCTT & (Roier et \\
\hline & AAGGCGTTTAAGGGCACCAATAA & \\
\hline \multirow[t]{2}{*}{ yrbE_5 } & TTATTGGTGCCCTTAAACGCCTTA & (Roier et \\
\hline & AGGATTTTTTTATGAGACAAACAA & 0) \\
\hline yrbE_6 & GCAGAGGTTGATTTGTAGTATGA & $\begin{array}{l}\text { (Roier et } \\
\text { al., 2016) }\end{array}$ \\
\hline IpsA_1 & AAGCCGACAGTAAAGTCTGGAT & This study \\
\hline \multirow[t]{2}{*}{ IpsA_2 } & TGTATGATGGTGTTTTTGAGGTG & This study \\
\hline & CATAGAAATTTTAACCGCACTTTT & \\
\hline \multirow[t]{2}{*}{ lpsA_3 } & AAAAGTGCGGTTAAAATTTCTAT & This study \\
\hline & GCACСТCAААААСАССАТСАТАСА & \\
\hline \multirow[t]{2}{*}{ IpsA_4 } & AAGATTTTTAACCGCACTTTTGTTA & This study \\
\hline & GTTTAAGGGCACCAATAACTGC & \\
\hline IpsA_5 & GCAGTTATTGGTGCCCTTAAAC & This study \\
\hline
\end{tabular}


TAACAAAAGTGCGGTTAAAAATCTT

IpsA_6

CAACCGCACTTTGATGTCGTG

This study

\begin{tabular}{|c|c|c|}
\hline \multicolumn{2}{|c|}{ Complementatio } & \\
\hline IpsA+_1 & AAGCCGACAGTAAAGTCTGGAT & This study \\
\hline \multirow[t]{2}{*}{ IpsA+_2 } & TCTTGTGCAATGTAACATCAGA & This study \\
\hline & ACATAGCCAAGTAAAATTTGAACTT & \\
\hline \multirow[t]{2}{*}{$\operatorname{lpsA}+3$} & AAGTTCAAATTTTACTTGGCTATGT & This study \\
\hline & TCTGATGTTACATTGCACAAGA & \\
\hline \multirow[t]{2}{*}{$\operatorname{lps} \mathrm{A}+\ldots 4$} & CACAACAAAAGGCAGTCCAGC & This study \\
\hline & TGAAGAAGGTGTTGCTGACTCA & \\
\hline \multirow[t]{2}{*}{ IpsA+_5 } & TGAGTCAGCAACACCTTCTTCA & This study \\
\hline & GCTGGACTGCCTTTTGTTGTG & \\
\hline $\operatorname{lps} A+\ldots 6$ & GCGATAGAGTGAAAGAAACGCT & This study \\
\hline \multicolumn{3}{|c|}{ MLST analysis $^{I}$} \\
\hline adk_up & GGTGCACCGGGTGCAGGTAA & \\
\hline adk_down & CCTAAGATTTTATCTAACTC & \\
\hline atpG_up & ATGGCAGGTGCAAAAGAGAT & \\
\hline atpG_down & TTGTACAACAGGCTTTTGCG & \\
\hline frdB_up & CTTATCGTTGGTCTTGCCGT & \\
\hline frdB_down & TTGGCACTTTCCACTTTTCC & \\
\hline fucK_up & ACCACTTTCGGCGTGGATGG & \\
\hline fucK_down & AAGATTTCCCAGGTGCCAGA & \\
\hline mdh_up & TCATTGTATGATATTGCCCC & \\
\hline mdh_down & ACTTCTGTACCTGCATTTTG & \\
\hline pgi_up & GGTGAAAAAATCAATCGTAC & \\
\hline pgi_down & ATTGAAAGACCAATAGCTGA & \\
\hline recA_up & ATGGCAACTCAAGAAGAAAA & \\
\hline recA_down & TTACCAAACATCACGCCTAT & \\
\hline
\end{tabular}

Taccording to Meats et al., 2003 
Figure 1: No observable correlation of serum resistance and invasiveness. Clinical isolates were tested for their resistance to $20 \%$ human serum. Invasive isolates $(n=46)$ and non-invasive isolates $(\mathrm{n}=31)$ do not show a significant difference in their survival rates $(P>0.05$, Mann-Whitney $U$ Test). The fluorescence levels are a measure of survival by indicating metabolic acivity. The heatinactivated serum control treated sample readout corresponds to $100 \%$ fluorescence signal. Shown is the median with interquartile range, measurement was done once with two technical replicates.

Figure 2: NTHi23 shows similar serum resistance when compared to the encapsulated strain Hib Eagan. The survival of the two strains was determined in different amounts of serum. Shown are the mean values and the standard deviation of three independent measurements, normalized to a $0 \%$ serum control. To note, no statistical significance can be obtained, e.g. using 2-way ANOVA with Bonferroni posttest for all time points tested.

Figure 3 Commonly identified serum resistance mechanisms can also be found in NTHi23. All three mutants (NTHi23 $\Delta$ siaP, $\Delta y r b E$ and $\triangle H i 0461$ ) show decreased survival in $20 \%$ human serum, when compared to the NTHi23 wt. Data were normalized to a heat-inactivated control. Median and interquartile range of at least five independent measurements are shown. Single asterisks mark significant differences $(P<0.05$; Unpaired t Test).

Figure 4: In vitro cultivation leads to the identification of a serum sensitive variant of NTHi23. The isolated variant strain of NTHi23 shows 1000-fold decreased serum resistance when compared to the original strain in $20 \%$ normalized human serum. Data were normalized to a heat-inactivated control. Median and interquartile range is indicated of at least four independent measurements. An asterisk marks significant differences $(P<0.05$; Mann-Whitney $U$ Test $)$. 
Figure 5: NTHi23 $\Delta l p s A$ confirms the role of the $\operatorname{lps} A$ phase-off switch in the serum sensitive phenotype of NTHi23_variant. NTHi23 wt, variant, $\Delta l p s A$ and $\Delta l p s A \operatorname{lps} A^{+}$were tested for survival in $20 \%$ normal human serum. Both, the $\operatorname{lps} A$ deletion and the $\operatorname{lps} A$ phase-off mutant (NTHi23_variant) show significantly decreased serum resistance when compared to the wt and show comparable survival rates when compared to each other. The complementation strain $(\Delta \operatorname{lps} A$ $\operatorname{lps} A^{+}$) reaches serum resistance levels as high as the wt strain. Shown is the median and interquartile range of four independent assays. Asterisks mark significant differences $(P<0.05$; Mann-Whitney $U$ Test).

Figure 6: The NTHi23 wt strain outcompetes $\Delta l p s A$ in the invasion of epithelial cells. NTHi23 wt and $\Delta l p s A$ were tested for adhesion and invasion of human epithelial cells. The wt and the mutant strain show no significant difference in adhesion to A549 cells (A). For the invasion assay, $\triangle l p s A$ shows significant attenuation (B). The assays were performed three times independently, with three technical replicates each. Shown is median and interquartile range. The asterisk marks a significant difference $(P<0.05$; Mann-Whitney $U$ Test $)$. 


\section{References}

Anderson, P., Johnston, R.B., Jr., Smith, D.H., 1972. Human serum activities against Haemophilus influenzae, type b. J. Clin. Invest. 51, 31-38.

Cerquetti, M., Cardines, R., Ciofi Degli Atti, M.L., Giufre, M., Bella, A., Sofia, T., Mastrantonio, P., Slack, M., 2005. Presence of multiple copies of the capsulation b locus in invasive Haemophilus influenzae type $\mathrm{b}(\mathrm{Hib})$ strains isolated from children with Hib conjugate vaccine failure. J. Infect. Dis. 192, 819-823.

Clark, S.E., Eichelberger, K.R., Weiser, J.N., 2013. Evasion of killing by human antibody and complement through multiple variations in the surface oligosaccharide of Haemophilus influenzae. Mol. Microbiol., 88, 603-618.

Clark, S.E., Snow, J., Li, J., Zola, T.A., Weiser, J.N., 2012. Phosphorylcholine allows for evasion of bactericidal antibody by Haemophilus influenzae. PLoS Pathog. 8, e1002521.

Corn, P.G., Anders, J., Takala, A.K., Kayhty, H., Hoiseth, S.K., 1993. Genes involved in Haemophilus influenzae type b capsule expression are frequently amplified. J. Infect. Dis.167, 356-364.

Deadman, M.E., Lundstrom, S.L., Schweda, E.K., Moxon, E.R., Hood, D.W., 2006. Specific amino acids of the glycosyltransferase LpsA direct the addition of glucose or galactose to the terminal inner core heptose of Haemophilus influenzae lipopolysaccharide via alternative linkages. J. Biol. Chem. 281, 29455-29467.

Erwin, A.L., Nelson, K.L., Mhlanga-Mutangadura, T., Bonthuis, P.J., Geelhood, J.L., Morlin, G., Unrath, W.C., Campos, J., Crook, D.W., Farley, M.M., Henderson, F.W., Jacobs, R.F., Mühlemann, K., Satola, S.W., van Alphen, L., Golomb, M., Smith, A.L., 2005. Characterization of genetic and phenotypic diversity of invasive nontypeable Haemophilus influenzae. Infect. Immun. 73, 5853-5863.

Erwin, A.L., Sandstedt, S.A., Bonthuis, P.J., Geelhood, J.L., Nelson, K.L., Unrath, W.C., Diggle, M.A., Theodore, M.J., Pleatman, C.R., Mothershed, E.A., Sacchi, C.T., Mayer, L.W., Gilsdorf, J.R., Smith, A.L., 2008. Analysis of genetic relatedness of Haemophilus influenzae isolates by multilocus sequence typing. J. Bacteriol. 190, 1473-1483.

Figueira, M.A., Ram, S., Goldstein, R., Hood, D.W., Moxon, E.R., Pelton, S.I., 2007. Role of complement in defense of the middle ear revealed by restoring the virulence of nontypeable Haemophilus influenzae siaB mutants. Infect. Immun. 75, 325-333.

Giufre, M., Cardines, R., Caporali, M.G., Accogli, M., D'Ancona, F., Cerquetti, M., 2011. Ten years of Hib vaccination in Italy: prevalence of non-encapsulated Haemophilus influenzae among invasive isolates and the possible impact on antibiotic resistance. Vaccine 29, 3857-3862.

Gromkova, R., Rowji, P., Koornhof, H., 1989. Induction of competence in nonencapsulated and encapsulated strains of Haemophilus influenzae. Current Microbiol. 19, 241-245.

Hallstrom, T., Blom, A.M., Zipfel, P.F., Riesbeck, K., 2009. Nontypeable Haemophilus influenzae protein $\mathrm{E}$ binds vitronectin and is important for serum resistance. J. Immunol. 183, 25932601.

Hallstrom, T., Jarva, H., Riesbeck, K., Blom, A.M., 2007. Interaction with C4b-binding protein contributes to nontypeable Haemophilus influenzae serum resistance. J. Immunol. 178, 63596366.

Hallstrom, T., Resman, F., Ristovski, M., Riesbeck, K., 2010. Binding of complement regulators to invasive nontypeable Haemophilus influenzae isolates is not increased compared to nasopharyngeal isolates, but serum resistance is linked to disease severity. J. Clin. Microbiol. 48, 921-927.

Hallstrom, T., Riesbeck, K., 2010. Haemophilus influenzae and the complement system. Trends Microbiol. 18, 258-265. 
Hallstrom, T., Trajkovska, E., Forsgren, A., Riesbeck, K., 2006. Haemophilus influenzae surface fibrils contribute to serum resistance by interacting with vitronectin. J. Immunol. 177, 430436.

Hallstrom, T., Zipfel, P.F., Blom, A.M., Lauer, N., Forsgren, A., Riesbeck, K., 2008. Haemophilus influenzae interacts with the human complement inhibitor factor H. J. Immunol. 181, 537-545. High, N.J., Deadman, M.E., Moxon, E.R., 1993. The role of a repetitive DNA motif (5'-CAAT-3') in the variable expression of the Haemophilus influenzae lipopolysaccharide epitope alpha Gal(1-4)beta Gal. Mol. Microbiol. 9, 1275-1282.

Kelly, D.F., Moxon, E.R., Pollard, A.J., 2004. Haemophilus influenzae type b conjugate vaccines. Immunol. 113, 163-174.

Kraiss, A., Schlor, S., Reidl, J., 1998. In vivo transposon mutagenesis in Haemophilus influenzae. Appl. Environ. Microbiol. 64, 4697-4702.

Langereis, J.D., Stol, K., Schweda, E.K., Twelkmeyer, B., Bootsma, H.J., de Vries, S.P., Burghout, P., Diavatopoulos, D.A., Hermans, P.W., 2012. Modified lipooligosaccharide structure protects nontypeable Haemophilus influenzae from IgM-mediated complement killing in experimental otitis media. mBio 3, e00079-00012.

Langereis, J.D., Weiser, J.N., 2014. Shielding of a lipooligosaccharide IgM epitope allows evasion of neutrophil-mediated killing of an invasive strain of nontypeable Haemophilus influenzae. mBio 5, e01478-01414.

Lichtenegger, S., Bina, I., Roier, S., Bauernfeind, S., Keidel, K., Schild, S., Anthony, M., Reidl, J., 2014. Characterization of lactate utilization and its implication on the physiology of Haemophilus influenzae. Int. J. Med. Microbiol. 304, 490-498.

Malinverni, J.C., Silhavy, T.J., 2009. An ABC transport system that maintains lipid asymmetry in the gram-negative outer membrane. Proc. Natl. Acad. Sci. USA 106, 8009-8014.

Meats, E., Feil, E.J., Stringer, S., Cody, A.J., Goldstein, R., Kroll, J.S., Popovic, T., Spratt, B.G., 2003. Characterization of encapsulated and noncapsulated Haemophilus influenzae and determination of phylogenetic relationships by multilocus sequence typing. J. Clin. Mircobiol. 41, 1623-1636.

Nakamura, S., Shchepetov, M., Dalia, A.B., Clark, S.E., Murphy, T.F., Sethi, S., Gilsdorf, J.R., Smith, A.L., Weiser, J.N., 2011. Molecular basis of increased serum resistance among pulmonary isolates of non-typeable Haemophilus influenzae. PLoS Patho. 7, e1001247.

Nizet, V., Colina, K.F., Almquist, J.R., Rubens, C.E., Smith, A.L., 1996. A virulent nonencapsulated Haemophilus influenzae. J. Infect. Dis. 173, 180-186.

Noel, G.J., Brittingham, A., Granato, A.A., Mosser, D.M., 1996. Effect of amplification of the Cap $\mathrm{b}$ locus on complement-mediated bacteriolysis and opsonization of type b Haemophilus influenzae. Infect. Immun. 64, 4769-4775.

Oka, A., Sugisaki, H., Takanami, M., 1981. Nucleotide sequence of the kanamycin resistance transposon Tn903. J. Mol. Biol. 147, 217-226.

Pittman, M., 1931. Variation and type specificity in the bacterial species Haemophilus influenzae. J. Exp. Med. 53, 471-492.

Poole, J., Foster, E., Chaloner, K., Hunt, J., Jennings, M.P., Bair, T., Knudtson, K., Christensen, E., Munson, R.S., Jr., Winokur, P.L., Apicella, M.A., 2013. Analysis of nontypeable Haemophilus influenzae phase-variable genes during experimental human nasopharyngeal colonization. J. Infect. Dis. 208, 720-727.

Roier, S., Zingl, F.G., Cakar, F., Durakovic, S., Kohl, P., Eichmann, T.O., Klug, L., Gadermaier, B., Weinzerl, K., Prassl, R., Lass, A., Daum, G., Reidl, J., Feldman, M.F., Schild, S., 2016. A novel mechanism for the biogenesis of outer membrane vesicles in Gram-negative bacteria. Nat. Commun. 7, 10515. 
Rosadini, C.V., Ram, S., Akerley, B.J., 2014. Outer membrane protein P5 is required for resistance of nontypeable Haemophilus influenzae to both the classical and alternative complement pathways. Infect. Immun. 82, 640-649.

Rubach, M.P., Bender, J.M., Mottice, S., Hanson, K., Weng, H.Y., Korgenski, K., Daly, J.A., Pavia, A.T., 2011. Increasing incidence of invasive Haemophilus influenzae disease in adults, Utah, USA. Emerg. Infect. Dis. 17, 1645-1650.

Severi, E., Randle, G., Kivlin, P., Whitfield, K., Young, R., Moxon, R., Kelly, D., Hood, D., Thomas, G.H., 2005. Sialic acid transport in Haemophilus influenzae is essential for lipopolysaccharide sialylation and serum resistance and is dependent on a novel tripartite ATP-independent periplasmic transporter. Mol. Microbiol. 58, 1173-1185.

Sutton, A., Schneerson, R., Kendall-Morris, S., Robbins, J.B., 1982. Differential complement resistance mediates virulence of Haemophilus influenzae type b. Infect. Immun. 35, 95-104.

Tchoupa, A.K., Lichtenegger, S., Reidl, J., Hauck, C.R., 2015. Outer membrane protein P1 is the CEACAM-binding adhesin of Haemophilus influenzae. Mol. Microbiol. 98, 440-455.

Tong, H.H., Blue, L.E., James, M.A., Chen, Y.P., DeMaria, T.F., 2000. Evaluation of phase variation of nontypeable Haemophilus influenzae lipooligosaccharide during nasopharyngeal colonization and development of otitis media in the chinchilla model. Infect. Immun. 68, 4593-4597.

Ulanova, M., 2013. Global epidemiology of invasive Haemophilus influenzae type a disease: Do we need a new vaccine? J. Vaccine 2013, 14.

Voges, M., Bachmann, V., Naujoks, J., Kopp, K., Hauck, C.R., 2012. Extracellular IgC2 constant domains of CEACAMs mediate PI3K sensitivity during uptake of pathogens. PloS One 7, e39908.

Weiser, J.N., Pan, N., McGowan, K.L., Musher, D., Martin, A., Richards, J., 1998. Phosphorylcholine on the lipopolysaccharide of Haemophilus influenzae contributes to persistence in the respiratory tract and sensitivity to serum killing mediated by C-reactive protein. J. Exp. Med. 187, 631-640.

Zola, T.A., Lysenko, E.S., Weiser, J.N., 2009. Natural antibody to conserved targets of Haemophilus influenzae limits colonization of the murine nasopharynx. Infect. Immun. 7 , 3458-3465. 
Fig.1

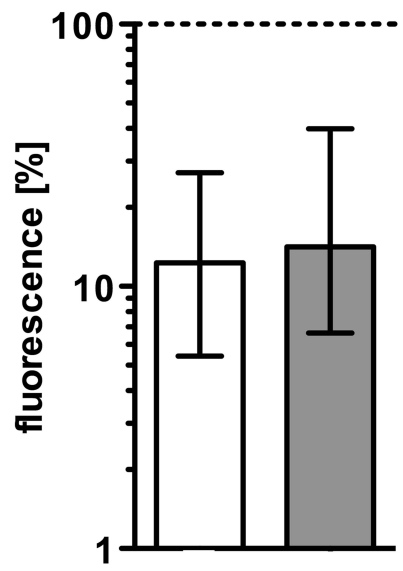

heat inactivated control

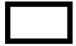

non-invasive $(n=31)$ invasive $(n=46)$

Normal Human Serum (20\%) 


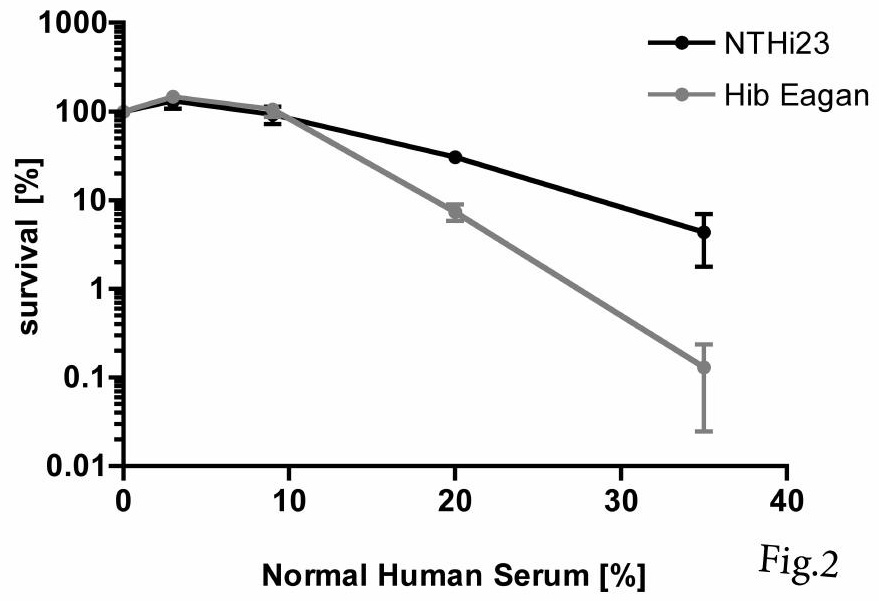




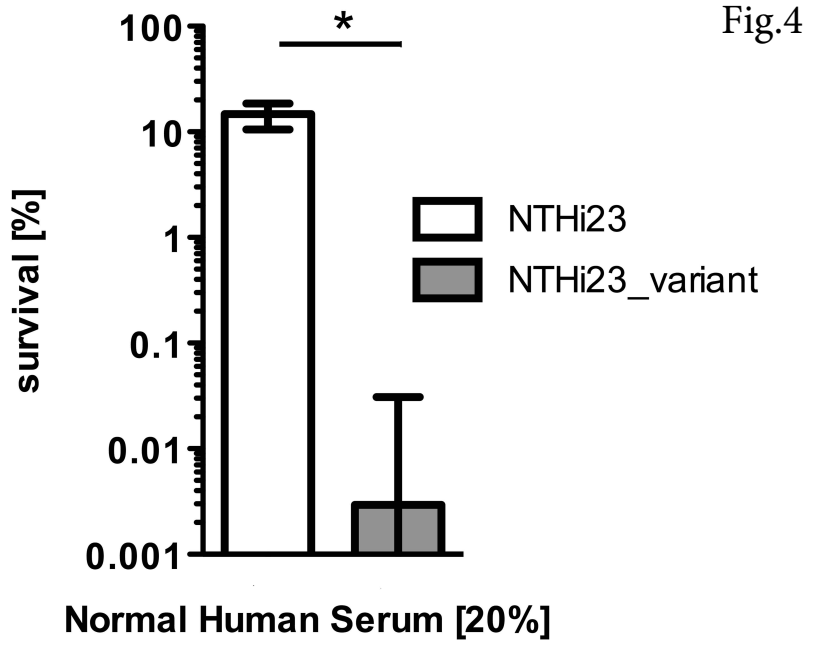




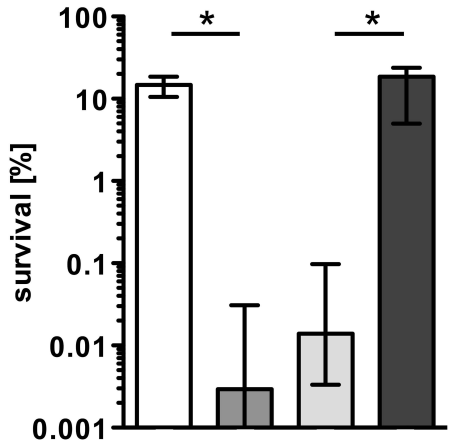

Fig.5

Normal Human Serum [20\%] 

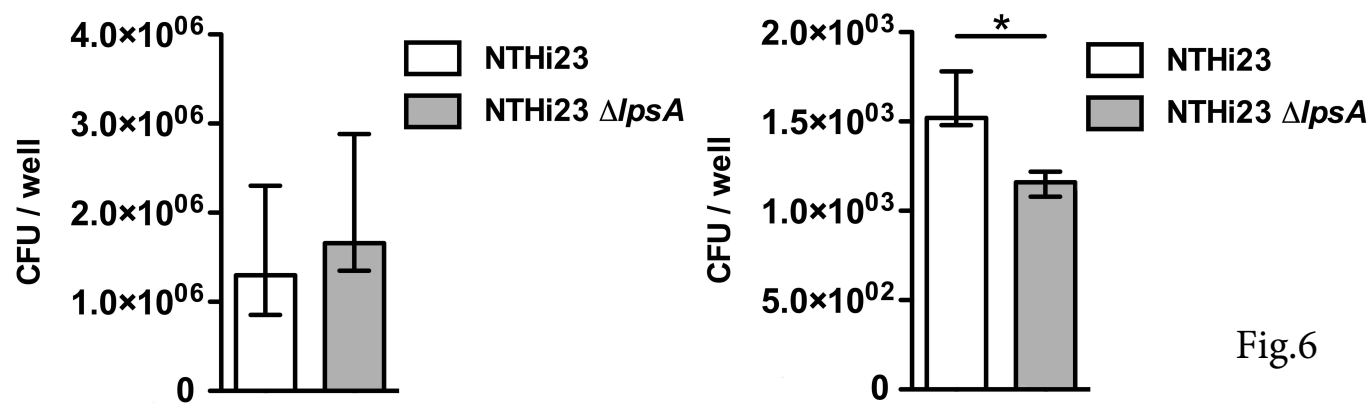